\title{
Genome-Wide Analysis of the NAC Gene Family in Physic Nut (Jatropha curcas L.)
}

\author{
Zhenying $\mathrm{Wu}^{1,2}$, Xueqin $\mathrm{Xu}^{1,2}$, Wangdan Xiong ${ }^{1,2}$, Pingzhi $\mathrm{Wu}^{1}$, Yaping Chen ${ }^{1}$, Meiru $\mathrm{Li}^{1}$, \\ Guojiang $\mathrm{Wu}^{1}$, Huawu Jiang ${ }^{1}$ *
}

1 Key Laboratory of Plant Resources Conservation and Sustainable Utilization, South China Botanical Garden, Chinese Academy of Sciences, Guangzhou 510650, China, 2 University of Chinese Academy of Sciences, Beijing 100049, China

* hwjiang@scbg.ac.cn

\section{G openaccess}

Citation: Wu Z, Xu X, Xiong W, Wu P, Chen Y, Li M, et al. (2015) Genome-Wide Analysis of the NAC Gene Family in Physic Nut (Jatropha curcas L.). PLoS ONE 10(6): e0131890. doi:10.1371/journal. pone.0131890

Editor: Jin-Song Zhang, Institute of Genetics and Developmental Biology, Chinese Academy of Sciences, CHINA

Received: April 18, 2015

Accepted: June 8, 2015

Published: June 30, 2015

Copyright: $\odot 2015$ Wu et al. This is an open access article distributed under the terms of the Creative Commons Attribution License, which permits unrestricted use, distribution, and reproduction in any medium, provided the original author and source are credited.

Data Availability Statement: All relevant data are within the paper and its Supporting Information files.

Funding: This work was supported by grants from the National Basic Research Program of China (973 Program) (grant no. 2010CB126603) (http://www.973. gov.cn/), and the Knowledge Innovation Program of the Chinese Academy of Sciences (grant no. KSCX2EW-J-28)(http://www.cas.cn/). The funders had no role in study design, data collection and analysis, decision to publish, or preparation of the manuscript.

\section{Abstract}

The NAC proteins (NAM, ATAF1/2 and CUC2) are plant-specific transcriptional regulators that have a conserved NAM domain in the $\mathrm{N}$-terminus. They are involved in various biological processes, including both biotic and abiotic stress responses. In the present study, a total of 100 NAC genes (JCNAC) were identified in physic nut (Jatropha curcas L.). Based on phylogenetic analysis and gene structures, $83 \mathrm{JcNAC}$ genes were classified as members of, or proposed to be diverged from, 39 previously predicted orthologous groups (OGs) of NAC sequences. Physic nut has a single intron-containing NAC gene subfamily that has been lost in many plants. The JCNAC genes are non-randomly distributed across the 11 linkage groups of the physic nut genome, and appear to be preferentially retained duplicates that arose from both ancient and recent duplication events. Digital gene expression analysis indicates that some of the JcNAC genes have tissue-specific expression profiles (e.g. in leaves, roots, stem cortex or seeds), and 29 genes differentially respond to abiotic stresses (drought, salinity, phosphorus deficiency and nitrogen deficiency). Our results will be helpful for further functional analysis of the NAC genes in physic nut.

\section{Introduction}

The NAC proteins are one of the largest groups of plant-specific transcriptional regulators, which share a conserved $\mathrm{N}$-terminus DNA binding domain originally found in four genes: ATAF1/ATAF2 and Cup-Shaped Cotyledon2 (CUC2) in Arabidopsis and No Apical Meristem $(N A M)$ in Petunia hybrida. In petunia, NAM is required for pattern formation in embryos and flowers and expressed at meristem and primordia boundaries [1]. Arabidopsis CUC1 and CUC2 are involved in the formation of embryo, floral organs and shoot apical meristems [2]. ATAF1 and ATAF2 are reported to respond to biotic and abiotic stress in Arabidopsis [3, 4]. NAC proteins contain a NAM domain consisting of five conserved subdomains at the $\mathrm{N}$-terminus (designated A-E) [5, 6]. Subdomains A, C and D are highly conserved, while B and E are more variable [7]. 
Competing Interests: The authors have declared that no competing interests exist.
NAC proteins have been found to participate in diverse plant developmental processes, including apical meristem development [1, 8], organ formation and development [9], leaf senescence [10], fruit ripening [11], hormone signaling [12], and both biotic and abiotic stress responses [12,13]. Accordingly, large numbers of putative NAC genes have been identified, including 117 in Arabidopsis and 151 in rice (Oryza sativa L.) [14], 74 in grape (Vitis vinifera) [15], 152 in soybean (Glycine max L.) [16], 88 in pigeonpea (Cajanus cajan (L.) Millsp.) [17], 147 in foxtail millet (Setaria italica L.) [18], and 204 in Chinese cabbage (Brassica pekinensis) [19]. Comprehensive analysis of the NAC family genes in rice and Arabidopsis has divided the NAC proteins into two large groups (Groups I and II), and sequences of the NAC domain in Group I, but not in Group II are highly conserved [7]. In addition, based on an expert NAC sequence comparison, 40 orthologous groups (OGs) of NAC sequences were proposed to be probably derived from an ancestral gene which presents in the most recent common ancestor of dicots and monocots [20].

Physic nut (Jatropha curcas L.) is a perennial plant of the Euphorbiaceae family that has received great attention as a renewable resource for biodiesel production. It has attractive features for its high resistance to various stresses, including drought and nutrient deficiencies [21]. Thus, its transcriptional responses to several stresses, including cold [22] and salinity [23], have been examined in attempts to elucidate the mechanisms involved in and (potentially) extend its resistance. However, the roles of NAC proteins in physic nut have not yet been explored, although they play key roles in the formation of plants' organs [9] and stress responses $[12,13]$.

Genomic sequence and expressed sequence tags (ESTs) libraries that we and others have recently constructed [24-26] provide convenience for us to analyze the gene families and their evolution in physic nut. For example, 58 WRKY genes were identified in the physic nut genome, and no evidence of recent gene duplication was detected in a phylogenetic comparison of JcWRKY genes and those of Arabidopsis, rice and castor bean [27]. In the study presented here, we identified the NAC genes of physic nut (JcNACs) from genome sequences. We then characterized the overall exon/intron arrangements of the full genes and the conserved NAC domain-coding sequences. The distribution of these genes in the linkage groups (LGs) and a phylogenetic tree combining castor bean, Arabidopsis and rice NAC proteins were also analyzed to examine their evolutionary relationships and the putative functions of physic nut NAC proteins. Finally, we analyzed the expression patterns of $J c N A C$ genes under both normal growth conditions and abiotic stresses.

\section{Materials and Methods}

\section{Ethics statement}

No specific permits were required for the field studies described because the experiment site is owned by South China Botanical Garden, Chinese Academy of Sciences, and the Key Laboratory of Plant Resources Conservation and Sustainable Utilization undertook the management. No specific permits were required for these locations/activities, because the location is not privately-owned or protected in any way and the field studies did not involve endangered or protected species.

\section{Sequence retrieval and identification}

Sequences of Arabidopsis NAC proteins were retrieved from the Arabidopsis genome database, TAIR 9.0 (http://www.arabidopsis.org/), and used as queries in BLAST searches against the physic nut genome database of the KaZuSa DNA Research Institute (http://www.kazusa.or.jp/ jatropha/) [24] and our own genome database, which is available from DDBJ/EMBL/GenBank 
under Accession no. AFEW00000000 (the version described in this paper is the first version, AFEW01000000). Sequences were selected for further analysis if the $\mathrm{E}$ value was less than $1 \mathrm{e}^{-10}$. Next, we corrected errors in annotation of NAC coding sequences on the basis of the physic nut EST database available from GenBank (http://www.ncbi.nlm.nih.gov/) and our own physic nut and Jatropha integerrima (allied species of physic nut) EST datasets (SRA197144 and SRA197148 in GenBank). Finally, all putative proteins were confirmed to be NAC proteins by the Pfam program (http://pfam.xfam.org/) and the confirmed sequences were aligned using Clustal $\mathrm{X}$ to remove redundant sequences. In addition, length, molecular weight and isoelectric point parameters of each JcNAC protein were calculated by the online ExPasy program (http:// www.expasy.org/tools/) [28].

\section{Chromosomal localization of the JcNAC genes}

Chromosome localization was completed using MapChart 2.1 [29] based on the linkage map we have constructed [26]. Firstly, the specific positions of each JcNAC gene on corresponding scaffold were obtained from our genome database. Secondly, the detailed locations of each $J c N A C$ gene were calculated based on the adjacent mapping markers located on our geneticlinkage map. Lastly, chromosome localization was completed using MapChart 2.1. Tandem repeats were defined as repeats located within $50 \mathrm{~kb}$ of each other, or separated by less than 4 non-homologous spacer genes [30].

\section{Phylogenetic analysis of the NAC gene family}

To analyze relationships of the NAC genes in physic nut, we used putative JcNAC proteins and NAC proteins from another three species to construct a phylogenetic tree: Arabidopsis (dicotyledon), rice (monocotyledon) and castor bean (dicotyledon, Euphorbiaceae). Sequences of the rice NAC proteins were downloaded from the rice genome annotation database (http://rice. plantbiology.msu.edu/, release 5.0). Castor bean NAC proteins were obtained from Phytozome (http://www.phytozome.net) [31]. Proteins of 28229.m000059, 28492.m000489, 30076. m004482 and 30157.m000796 were not used for alignments and phylogenetic analysis because sequences of their NAC domains are incomplete. Multiple sequence alignments of the conserved NAC domain sequences were performed using Clustal W. Unrooted maximum-likelihood (ML) trees were constructed using the LG model with aLRT SH-like branch support steps in PhyML version 3.0 (http://atgc.lirmm.fr/phyml/) [32].

\section{Gene structure analysis and conserved motif composition prediction}

The exon/intron structures of $J c N A C$ genes were determined by comparing the coding sequences and corresponding genomic sequences in the Gene Structure Display Server (GSDS, http://gsds.cbi.pku.edu.cn/) [33]. Conserved motifs were analyzed using the MEME program (http://meme.nbcr.net/meme/) [34]. Transmembrane helices in JcNAC proteins were predicted using TMHMM Server v. 2.0 (http://www.cbs.dtu.dk/services/TMHMM-2.0/) [35]. The cis-elements analysis was performed in the promoter sequences ( $1 \mathrm{~kb}$ upstream region) of the JcNAC coding main sequences using the program MEME [34] and PLACE (A Database of Plant cis-acting Regulatory DNA Elements, http://www.dna.affrc.go.jp/PLACE/signalscan. html) [36]. The MEME program was used to search new putative cis-motif consensus patterns of 8-12 bases width, with E-value less than 0.01 , only on the forward strand of the input sequences. The PLACE program was used to search the known cis-acting elements reported in the previous studies, which commonly existed in all of the corresponding JcNAC genes' promoters. 


\section{Preparation of plant materials}

After disinfection with 1:5000 $\mathrm{KMnO}_{4}$ solution, seeds of the inbred physic nut (J. curcas L.) cultivar GZQX0401 were planted in sand to germinate. When cotyledons were fully expanded, seedlings were transferred to trays containing a 3:1 mixture of sand and soil soaked with halfstrength Hoagland solution in a greenhouse $\left(30-35^{\circ} \mathrm{C}\right)$ illuminated with natural sunlight $(100$ $\left.300 \mu \mathrm{mol} \mathrm{m} \mathrm{s}^{-1}\right)$ in Guangzhou $\left(113.3^{\circ} \mathrm{E}, 23.1^{\circ} \mathrm{N}\right)$. After emergence of the first true leaf, the trays were irrigated with 1 liter of Hoagland nutrient solution ( $\mathrm{pH}$ 6.0) once every two days at dusk. The Pi and $\mathrm{N}$ deficiency treatment was begun at the six-leaf stage (eight weeks after germination), after removing most nutrients by five washes with 1 liter of tap water. The plants assigned to the $\mathrm{P}$ - or $\mathrm{N}$ - deficiency treatments were then irrigated daily with Hoagland nutrient solution minus phosphorus or minus nitrogen). For the salinity treatment, the seedlings were irrigated with Hoagland solution plus $100 \mathrm{mM} \mathrm{NaCl}$ every day. For the drought treatment, irrigation was withheld. Roots were sampled after 1 day, 4 days and 7 days of drought stress; after 2 hours, 2 days and 7 days of salinity stress; and after 2 hours and 2 days of phosphorus and nitrogen deficiency. Samples were frozen immediately in liquid nitrogen and stored at $-80^{\circ} \mathrm{C}$ prior to quantitative PCR (qRT-PCR) analysis.

\section{RNA isolation and qRT-PCR analysis}

Total RNA of roots was extracted using the CTAB method with some modifications [37]. Briefly, the CTAB extraction buffer was spermidine-free, and $1 / 4$ volume $5 \mathrm{M} \mathrm{KAc}(\mathrm{pH} 4.8)$ was added before the first centrifugation; $1 / 4$ volume $8 \mathrm{M} \mathrm{LiCl}$ was used for precipitation; and the pellet was dissolved in guanidine thiocyanate solution ( $2.5 \mathrm{~g}$ guanidine thiocyanate in 6.6 $\mathrm{mL}$ CBS solution ( $42 \mathrm{mM}$ sodium citrate and $8.38 \mathrm{~g} \mathrm{~L}^{-1} \mathrm{~N}$-lauroylsarcosine sodium) instead of SSTE. Subsequently, first-strand cDNA was synthesized from $2 \mu \mathrm{g}$ of total RNA treated with RNase-Free DNase I (Roche), using M-MLV reverse transcriptase (Promega) according to the manufacturer's instructions, and qRT-PCR analysis was performed using a physic nut Actin gene as an internal control. The specific primer sequences and the PCR reaction conditions are listed in S1 Table. A LCS480 system (Roche) was used for all qRT-PCR assays. Each $20 \mu \mathrm{L}$ reaction volume included $10 \mu \mathrm{L}$ of twofold SYBR Premix ExTaq, $0.4 \mu \mathrm{L}$ of forward and reverse primer $(10 \mu \mathrm{mol}), 2 \mu \mathrm{L}$ of diluted cDNA solution, and $7.2 \mu \mathrm{L}$ of $\mathrm{ddH}_{2} \mathrm{O}$ The thermal profile used for all PCR amplifications was: $10 \mathrm{~min}$ at $95^{\circ} \mathrm{C}$ for DNA polymerase activation, followed by 40 cycles of $15 \mathrm{~s}$ at $95^{\circ} \mathrm{C}$ and $35 \mathrm{~s}$ at $60^{\circ} \mathrm{C}$. The expression levels were calculated using the 2 $\triangle \triangle C T$ method. Each PCR assay was run in triplicate for two independent biological repeats.

\section{Results}

\section{Identification and characterization of JcNAC genes}

Based on the domain sequences of the NAC family members of Arabidopsis and rice, a total of 100 NAC family members were identified both from the publicly available genome database [24] and our physic nut database (DDBJ/EMBL/GenBank under accession no.

AFEW00000000) [26] using the BlastP and tBlastn programs. Next, we corrected errors in several annotated NAC coding sequences according to the available EST sequences for physic nut in GenBank and our physic nut and J. integerrima L. EST database (SRA197144 and SRA197148 in GenBank). We designated JcNAC001 to JcNAC100 according to the phylogenetic tree constructed from alignments of predicted NAC proteins, and their accession numbers in GenBank, and parameters of the predicted proteins, are listed in S2 Table. According to the detailed information, lengths of these predicted JcNAC proteins ranged from 147 (JcNAC070) to 709 residues (JcNAC066), with an average of 340 aa, and the isoelectric point 
(PI) ranged from 4.56 (JcNAC077) to 9.56 (JcNAC060). To analyze the NAM domain of these predicted JcNAC proteins, the Pfam program was used and the results indicated that they indeed contained a conserved NAM domain (pfam02365/cl03558) $[5,6]$ at the N-terminus of the amino acid sequences (S2 Table).

Several NAC proteins have been previously shown to own a membrane-bound domain [38]. In this study, the membrane-bound domain of all the JcNAC proteins was analyzed using the TMHMM server v. 2.0. Eight JcNAC proteins (JcNAC044, 047, 049, 050, 054, 055, 071 and 074) were observed to contain transmembrane amino acid sequences in their C-terminus (S2 Table). Analysis of exon/intron structures displayed that 58 JcNAC genes have two introns, 10 have one intron, 10 have five introns, 8 have three introns, 7 have four introns, and 7 genes are intronless within coding sequences in their ORF (open reading frame). Nine genes have intron in the untranslated region (Fig 1A).

\section{Conserved motifs of the JcNAC proteins}

Analysis of the sequence features of JcNAC proteins using MEME [34] predicted 10 conserved motifs (S1 Fig). Amino acid sequences of motifs 1 plus 8, 4, 6 plus 2, 5 plus 3, and 7 respectively correspond to the five well-conserved subdomains (A-E) of the NAC domain [39]. Motif 9 is similar to motif 6 plus 2, composing subdomain C (Fig 1B). The amino acid sequences of these motifs are highly conserved, like homologous sequences detected in NAC proteins from Arabidopsis, pigeonpea, rice, and Populus [7, 17, 40]. Motif 10 corresponds to a C-terminus transcription regulatory region (TRR). About $60 \%$ of the predicted JcNAC proteins contain all five characteristic motifs of the NAC domain, while the others appear to have lost one or more of these motifs.

The exon/intron structures of NAC domain coding sequences have been shown to be conserved in grape, Arabidopsis, rice, and banana (Musa acuminate) NAC genes, and the introncontaining genes have been classified into nine models in the previous studies [15, 20]. Analysis of the overall exon/intron arrangements showed that intron-containing NAC genes from physic nut could be grouped into these model structures of NAC domain-coding sequences as well (S2 Table). The NAC domain of 73 intron-containing genes in physic nut was encoded by the first three exons: the first encoding the subdomains $\mathrm{A}$ and $\mathrm{B}$, the second encoding the subdomains $\mathrm{C}$ and $\mathrm{D}$, and the third encoding the subdomain $\mathrm{E}$ (S2 Table). Among these members, $J c N A C 046$ and JcNAC061 have lost the first intron, while JcNAC042 has lost both introns. The NAC domain in JcNAC062-065 is encoded by the second to the fourth exons (the intron between the third and fourth exons is missing in JcNAC063 and 067). While in JcNAC094-096, it is encoded by the second and third exons, and the second exon encodes the subdomains A and B, while the third exon encodes the subdomains C-E. In JcNAC092 and 093, it is encoded by the third and fourth exons. In the single intron-containing genes, JcNACO78 and JcNAC082090, the first exon encodes the subdomains A-D, and the second exon encodes the subdomain E (S2 Table).

\section{Phylogenetic analysis of NAC proteins}

The previous phylogenetic analysis has classified the NAC family genes into two major groups, Groups I and II, and those of Group I (but not Group II) are highly conserved [7]. Group I and some Group II members have been further classified into 40 orthologous groups (OGs) for dicots and monocots [20]. To investigate the phylogenetic relationships among the NAC genes in physic nut and other plants, a Maximum Likelihood phylogenetic tree was constructed in PhyML version 3.0 [32] from alignments of the conserved NAC domain sequences of all predicted NAC proteins from physic nut (dicot, Euphorbiaceae) and castor bean (dicot, 
(A)

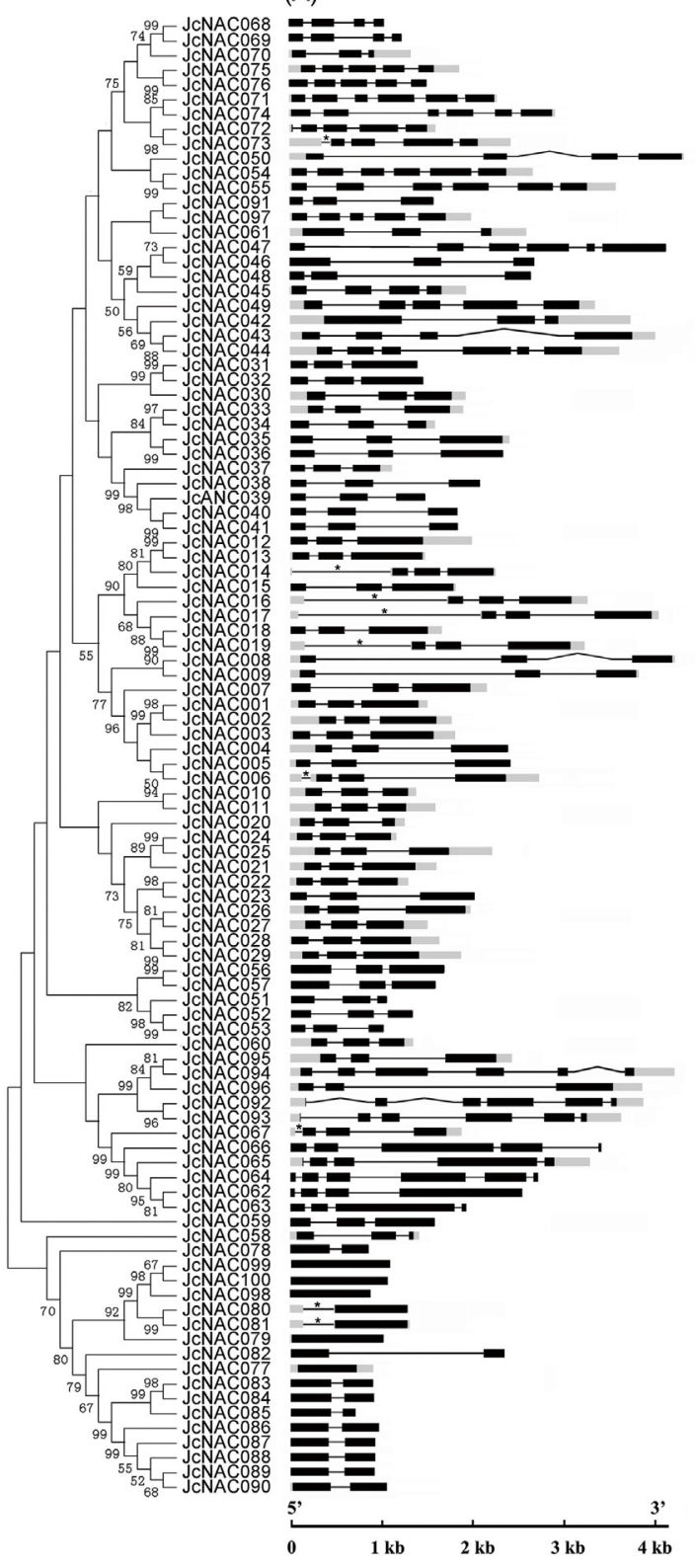

(B)

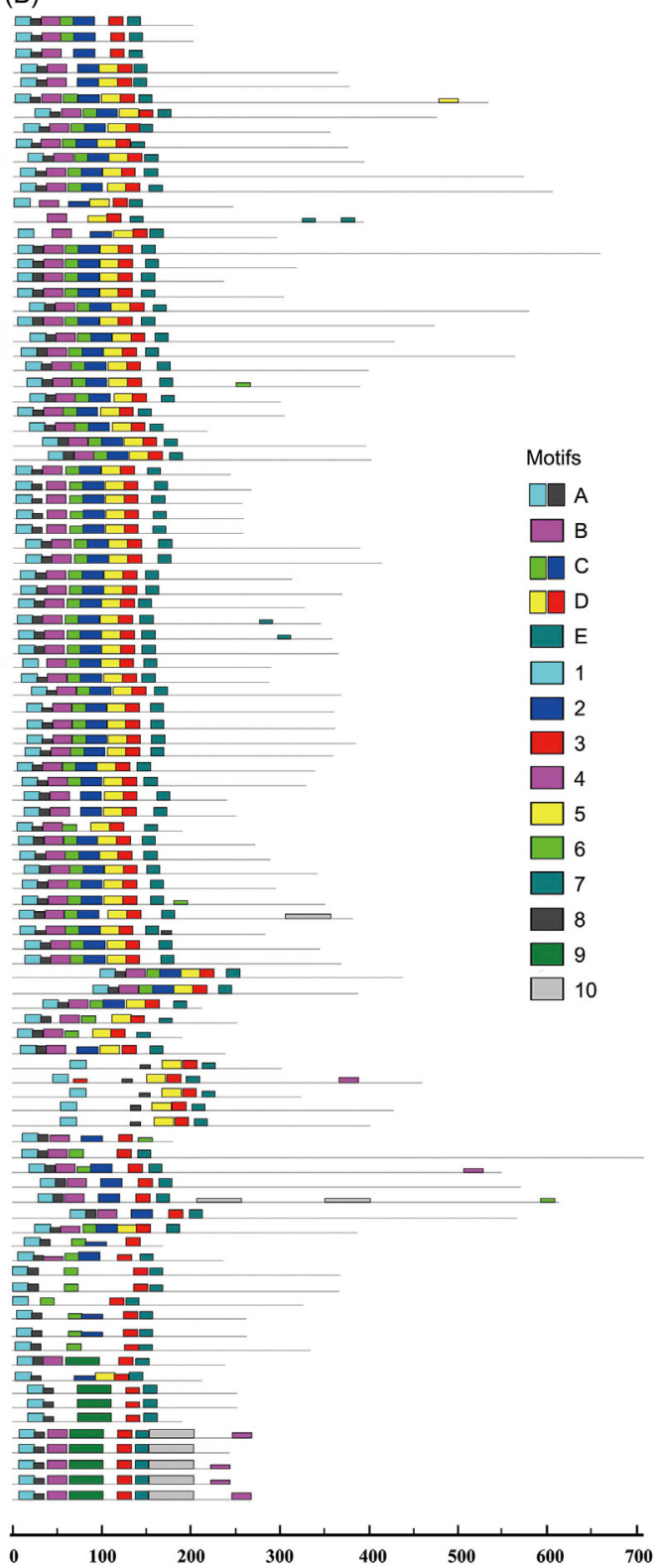

Fig 1. Gene structure and motif locations of JcNAC genes. (A), Exon/intron arrangements of JcNAC genes. Exons and introns are represented by black boxes (open reading frame in black, untranslated region (UTR) in gray), and black lines, respectively, and their sizes are indicated by the scale at the bottom. *, intron in the UTR. (B), Schematic representation of conserved motifs in the JcNAC proteins predicted by MEME. Each motif is represented by a number in the colored box. The black lines represent non-conserved sequences. The relationships between motifs and conserved domains (A-E) are shown on the right. The lengths of JcNAC proteins can be estimated from the scale at the bottom.

doi:10.1371/journal.pone.0131890.g001

Euphorbiaceae), and partial NAC proteins (including all proteins that had been classified into OGs) [20] from rice (monocot) and Arabidopsis (dicot) (S2 Fig). This phylogenetic tree was perfectly consistent with the determined OGs of NAC genes for grape, Arabidopsis, rice and banana [20] when compared the genes from Arabidopsis and rice in each of the subclusters. The phylogenetic tree clarified that these NAC proteins could be divided into the 6 main clusters (CL00002-00007) determined by Cenci et al. [20]. So, we also performed six additional phylogenetic analyses with the orthologous genes of each main cluster to verify their phylogenetic 
Table 1. Distribution of physic nut NAC genes in orthologous groups (OGs).

\begin{tabular}{|c|c|c|c|c|c|c|c|}
\hline OGs & Genes & OGs & Genes & OGs & Genes & OGs & Genes \\
\hline $1 a$ & JcNACOO9 & $3 d$ & JcNAC029 & \multirow[t]{13}{*}{$5 \mathrm{bL}$} & JcNAC064 & $8 a$ & JcNAC034 \\
\hline $1 b$ & JcNAC008 & $3 e$ & JcNAC021 & & JcNAC065 & \multirow[t]{5}{*}{$8 b$} & JcNAC037 \\
\hline $1 c$ & JcNAC006 & \multirow[t]{6}{*}{$3 L$} & JcNAC051 & & JcNAC066 & & JcNAC038 \\
\hline $1 d$ & JcNACOO4 & & JcNAC052 & & JcNAC067 & & JcNAC039 \\
\hline $1 e$ & JcNAC007 & & JcNAC053 & & JcNAC068 & & JcNAC040 \\
\hline $1 f$ & JcNAC005 & & JcNAC056 & & JcNAC069 & & JcNAC041 \\
\hline $1 \mathrm{~g}$ & JcNACOO3 & & JcNAC057 & & JcNAC070 & \multirow[t]{10}{*}{ SI } & JcNAC078 \\
\hline $1 \mathrm{~h}$ & JcNAC001 & & JcNAC059 & & JcNAC071 & & JcNAC082 \\
\hline & JcNACO02 & $4 a$ & JcNAC047 & & JcNAC072 & & JcNAC083 \\
\hline 1L & JcNAC091 & $4 b$ & JcNAC048 & & JcNAC073 & & JcNAC084 \\
\hline \multirow[t]{2}{*}{$2 a$} & JcNAC018 & $4 c$ & JcNAC045 & & JcNACO74 & & JcNAC085 \\
\hline & JcNAC019 & \multirow[t]{2}{*}{$4 d$} & JcNAC043 & & JcNAC075 & & JcNAC086 \\
\hline $2 b$ & JcNAC017 & & JcNAC044 & & JcNAC076 & & JcNAC087 \\
\hline $2 c$ & JcNAC016 & $4 e$ & JcNAC042 & \multirow[t]{2}{*}{$6 a$} & JcNAC035 & & JcNAC088 \\
\hline $2 d$ & JcNAC015 & $4 f$ & JcNAC046 & & JcNAC036 & & JcNAC089 \\
\hline $2 e$ & JcNAC014 & $4 g$ & JcNAC049 & \multirow[t]{2}{*}{$6 b$} & JcNAC031 & & JcNAC090 \\
\hline \multirow[t]{2}{*}{$2 f$} & JcNAC012 & \multirow[t]{4}{*}{$5 a$} & JcNAC010 & & JcNAC032 & IL1 & JcNAC077 \\
\hline & JcNAC013 & & JcNAC011 & $6 c$ & JcNAC0ЗО & \multirow[t]{6}{*}{ IL2 } & JcNAC079 \\
\hline \multirow[t]{2}{*}{$3 a$} & JcNAC024 & & JcNAC060 & \multirow[t]{2}{*}{$7 a$} & JcNAC095 & & JcNAC080 \\
\hline & JcNAC025 & & JcNAC058 & & JcNAC096 & & JcNAC081 \\
\hline $3 b$ & no & \multirow[t]{2}{*}{$5 b$} & JcNAC054 & $7 b$ & JcNAC094 & & JcNAC098 \\
\hline \multirow[t]{4}{*}{$3 c$} & JcNAC022 & & JcNAC055 & $7 c$ & JcNAC092 & & JcNAC099 \\
\hline & JcNAC023 & $5 c$ & JcNAC050 & $7 d$ & JcNAC093 & & JcNAC100 \\
\hline & JcNAC026 & $4 / 5 \mathrm{~L}$ & JcNAC061 & $7 e$ & JcNAC020 & & \\
\hline & JcNAC027 & \multirow[t]{2}{*}{$5 \mathrm{bL}$} & JcNAC062 & $7 f$ & JcNAC097 & & \\
\hline $3 d$ & JcNAC028 & & JcNAC063 & $8 a$ & JcNAC03З & & \\
\hline
\end{tabular}

doi:10.1371/journal.pone.0131890.t001

relationship (Panels A-F in S3 Fig). Based on these phylogenetic trees and their gene structures, $60 J c N A C$ genes were classified into the proposed 39 OGs of dicot NACs [20] with aLRT values spanning between 0.600 and 0.999 (except OG2f) (Table 1; S3 Table and Panels A-F in S3 Fig). Physic nut has multiple copies of genes in 14 of the 39 OGs. Among the 37 OGs which contain both dicot and rice genes, 30 showed the expected dicot and rice clustering in these phylogenetic analyses. ANAC105 in OG2b, ANAC045 and ANAC086 in OG4a, ANAC013 in OG4g, and 29992.m001406 in OG7e occupy unexpected positions. Three OGs (2a, 3c, and 5a) should contain gene duplications that occurred before the dicot and rice divergence.

Six JcNAC genes close to sequences of cluster 3 were classified as OG3-like (3L). These proteins share higher amino acid identities to OG3 proteins than other OG proteins, and they are encoded by two-intron containing genes. Fifteen $J c N A C$ genes close to cluster $5 \mathrm{~b}$ were classified as $5 \mathrm{bL}$. These genes have variable numbers of exons and introns, but their NAC domains share the highest amino acid identities with OG5 proteins, and are located in three exons (except for JcNAC063 and 067, which have lost the second intron). Two (JcNAC071 and 074) have a membrane-bound domain in the C-terminal region, which is the feature of several OGs4 and OGs5 proteins. Single intron containing genes (JcNAC078 and 082-090) were classified as SI, which have no detected orthologous genes in the castor bean genome.

The intronless (IL) genes were classed into two groups, named IL1 and IL2 (Table 1 and S3 Table). Proteins in the SI, IL1, and IL2 groups appeared to be very divergent from the other 
NACs. JcNAC061 and JcNAC091 also appeared to be very divergent from the other NACs. Based on the exon/intron structures, JcNAC061 and its orthologs (XP_002282899 in grape and $30170 . m 013849$ in castor bean) have two introns in their CDS, and NAC subdomains A-D are encoded by the first exon, suggesting that these genes have lost the first intron which present in most NAC genes. Their gene structures are similar to partial genes of OGs 4 and 5, so we named them as 4/5L. JcNAC091 was classified as $1 \mathrm{~L}$, based on the phylogenetic analysis and exon/intron structures, which is similar to OG1 genes (Table 1 and S2 Fig).

To examine the phylogenetic relationship among the NAC genes those were not classified into the $40 \mathrm{OGs}$ in physic nut, Arabidopsis and rice, an unrooted tree was constructed from alignments of the NAC domain sequences of these proteins (Panel G in S3 Fig). Based on this phylogenetic tree, the $5 \mathrm{bL}$ subgroup of physic nut was clustered into a group with the ANAC001 subgroup of Arabidopsis [7]. The intronless NAC genes from rice and Arabidopsis, and the IL and SI genes from physic nut were clustered into species specific subgroups, which indicated that they are expanded within different linkages and highly divergent from each other. The other genes contain one or two introns within their NAC domain coding sequences. The amino acid sequences of their NAC domains between different clades are highly divergent with low identities less than $40 \%$.

\section{Chromosomal distribution and duplication analysis of JcNAC genes}

A total of $98 J c N A C$ genes were mapped to 11 chromosomes according to the linkage map we constructed [26], while the other two genes (JcNAC024 and 064) located on unmapped scaffolds (S2 Table). The distribution and density of the JcNAC genes on chromosomes were not uniform (Fig 2). Chromosome 8 contained the highest frequency (21\%) of JcNAC genes, while chromosome 1, 3 and 5 contained the lowest frequencies (4\%). Evidence of both segmental and tandem duplication events of NAC genes in plants has been previously reported [14-16, 40]. To determine the possible relationships between the NAC genes and potential segmental duplications, we mapped the $J C N A C$ genes to the duplicated blocks established in the recent study [26]. As a result, five blocks of pairs (A1-A5) of $J c N A C$ genes were observed that may have arisen from the corresponding duplicate genomic blocks (Fig 2). In addition, a total of 30 $J c N A C$ genes (30\% of the total number) were observed to be in tandem repeats genes, which we designated them as T1-T11 (Fig 2). Nine direct tandem repeats (JcNAC065-069 and 071-074) (T7) were observed on chromosome 8 , which has the highest number of tandem repeats of $J c N A C$ genes. Their orthologs in castor bean were also clustered on a genomic sequence of scaffold 30076 (Fig 3). According to the phylogenetic tree, T4, T8, T9, and T11 genes duplicated after separation of lineages of the analyzed species; T6 genes (JcNAC003 and 026, originating from OGslg and 3c) on chromosome 6 arose from ancient tandem repeats, predating the divergence between monocots and dicots [20]; and T1 genes on chromosome 6 (JcNAC021 and 028 , originating from OGs $3 \mathrm{e}$ and $3 \mathrm{~d}$ ) predate the divergence of dicots because homologous repeats are present in genomes of grape (VvNAC17/GSVIVT01014403001 and VvNAC17/ GSVIVT01014405001) and Arabidospis (ANAC018/AT1G52880 and ANAC019/AT1G52890).

\section{Analysis of JcNAC genes' expression patterns}

After analyzing the EST (Expressed Sequence Tags) databases of physic nut and/or its related species J. integerrima, ESTs representing 80 of the $100 J c N A C$ genes were detected. Weak expression of six other $J c N A C$ genes was detected in our transcriptome expression database, generated using next-generation sequencing-based digital gene expression tags (S4 Table). Thus, 86 of the 100 predicted genes ( $86 \%$ of the total) were expressed in at least one of the tested tissues. To analyze the expression patterns of $J c N A C$ genes, we retrieved information on 

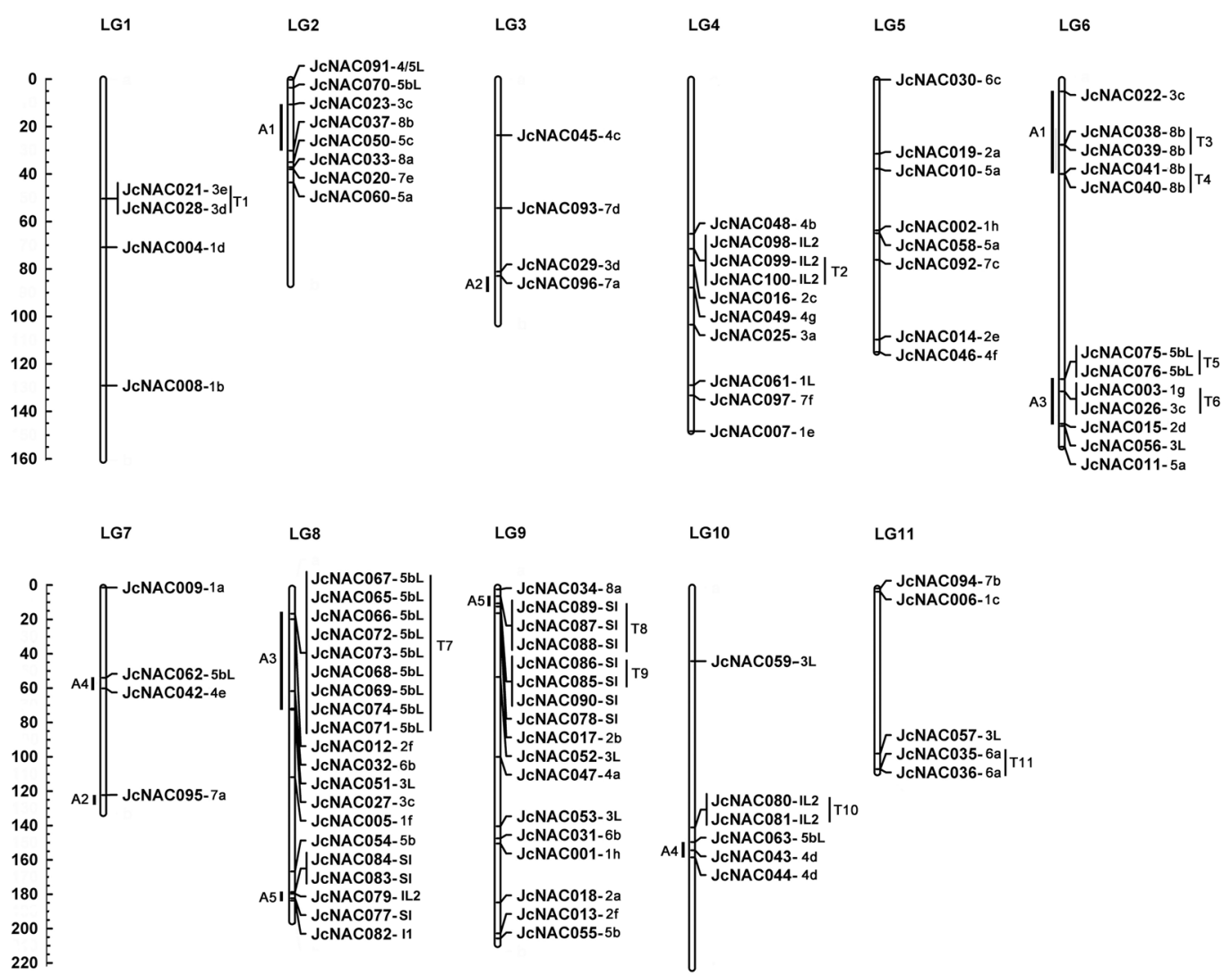

Fig 2. Chromosomal localization of physic nut NAC genes. Chromosomal localization of JCNAC genes based on the linkage map. In total, 98 JCNAC genes were mapped to the 11 linkage groups (LGs). The scale is in centimorgan. T, tandem duplication; A, ancient segmental duplication based on the genome synteny [26].

doi:10.1371/journal.pone.0131890.g002

expression levels of $J c N A C$ genes under normal growth conditions in four tissues: roots, stems (shoot cortex), leaves, and seeds (in the early development stage, S1, and filling and maturation stage, S2) [41]. Eleven of these genes were expressed in all tested tissues at relatively high levels (greater than or equal to 10 transcripts per million tags, TPM), namely JcNAC011, 021, 025,

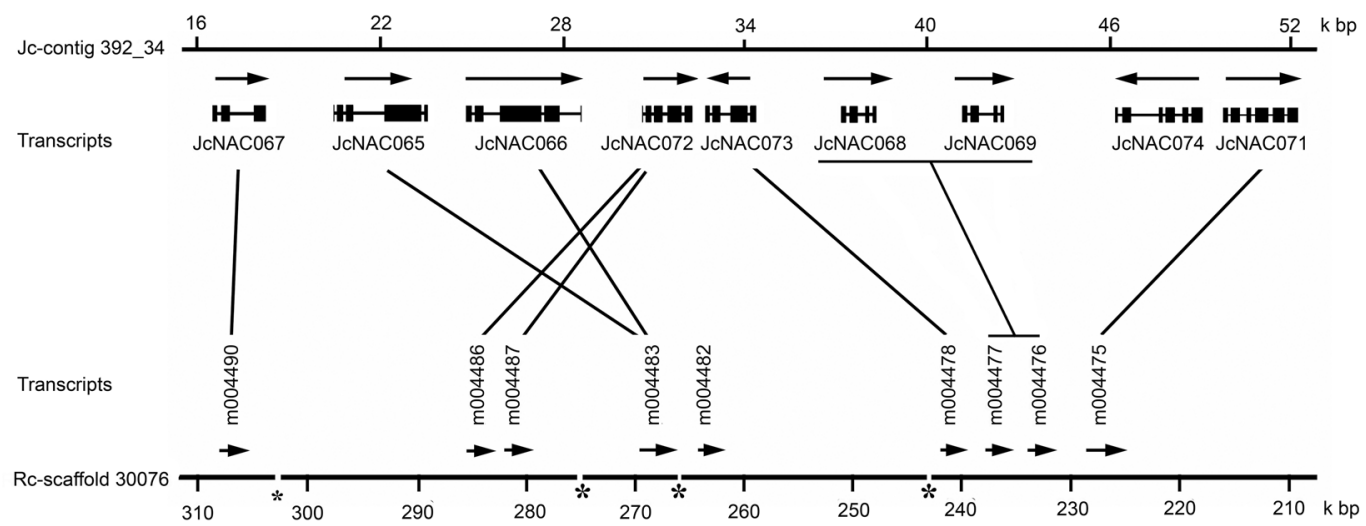

Fig 3. Synteny between physic nut and castor bean genomic segments containing NAC gene clusters. Nine genes in tandem repeats 7 (T7) on JCcontig 392 34 of physic nut and their orthologs on scaffold 30076 of castor bean were syntenic. The NAC domain of the 30076 .m004482 protein is incomplete. The orthologous genes based on the phylogenetic analysis (S2 Fig) were indicated by lines.

doi:10.1371/journal.pone.0131890.g003 
$043,044,049,054,055,067,073$, and 092 . However, many JcNAC genes were found to be expressed at different levels in different tissues according to the digital gene expression tags (Fig 4). In developing seeds, expression levels of five genes (JcNAC003, 021, 029, 050 and 094) were three-fold higher in the filling and maturation stage (S2) than in the early development stage (S1), while at least nine genes (JcNAC002, 011, 033, 037, 054, 060, 070, 092 and 097) were more strongly expressed at S1 than at S2 (S4 Table). Most of the single intron (SI) genes did not show any credible expression based on EST and digital gene expression tags in the above tissues in physic nut (Fig 4). ESTs of JcNAC082, 083, 087 and 090 orthologs were detected at high frequency in the J. integerrima flower cDNA library.

Several duplicators from genome triplication events in ancient dicotyledons were differently expressed among the tested tissues. For genes on the A1 locus, the middle expression level of $J c N A C 022$ (OG3c) in roots and JcNAC037 (OG8b) in S1 were observed, whereas JcNAC023 (OG3c) and JcNAC038-040 (OG8b) genes were not detected in the tested tissues. The $J c N A C 095$ gene (OG7a) but not the JcNAC096 gene (OG7a) on locus A2 was highly expressed in roots. The JcNAC026 gene (OG3c) on locus A3 was highly expressed in roots and leaves, while the JcNAC027 gene (OG3c) showed high expression level only in roots. For the tandem duplicators, the EST and high expression level of JcNAC035 (OG6a) were observed in roots, whereas JcNAC036 (OG6a) was not detected in the tissues tested on locus T11. For the 9 OG5bL genes on locus T7, the high expression level in the tested tissues was only observed for the JcNAC073 gene.

Next, we examined responses of $J_{c N A C}$ genes to four abiotic stresses (drought [42], salinity [23], phosphorus deficiency, and nitrogen deficiency) according to data retrieved from the next-generation sequencing-based digital gene expression tag database (two repeats). Differentially expressed genes (DEGs), with a greater than 2 -fold difference in expression and TPM greater than or equal to 5 at one time point (and $p$ value less than 0.01 ) were detected using Audic's algorithm with Bonferroni Correction (http://telethon.bio.unipd.it/bioinfo/IDEG6_ form/index.html) $[43,44]$. Twenty-nine JcNAC genes were found to respond to tested abiotic stresses according to these criteria (S4 Table). Of these 29 genes, most in OG3 reportedly respond to abiotic stresses in Arabidopsis and rice (S3 Table) [18]. The result of our analysis indicated that six of the $J c N A C$ genes in OG3 responded to the tested abiotic stresses in physic nut. To verify the digital gene expression (DGE) tag data, we selected some OG3 genes to examine the expression levels in roots after onset of drought, salinity, phosphorus and nitrogen deficiency stresses by qRT-PCR analysis (Fig 5). The results showed that JcNAC021, 024, 026 and 027 genes share the same expression pattern under both drought and salinity stresses. In addition, these five genes $(J c N A C 021,024,025-027)$ were all upregulated under phosphorus deficiency, but downregulated (except JcNAC024) under nitrogen deficiency in 2 days' roots (Fig 5). These results were generally consistent with expression changes observed in the digital gene expression tag profiling experiments.

The cis-acting elements control gene expression pattern in different tissues or stresses. So we analyzed the promoter regions ( $1 \mathrm{~kb}$ upstream) of all different expressed $J c N A C$ genes in each treatment (drought, salinity, phosphorus deficiency, and nitrogen deficiency). The results from the PLACE program gave the known cis-elements which were reported in previous studies [36] (S5 Table). For example, the S000407 is the dehydration responsive element [45] presented in the regulated genes in roots exposed to drought stress (Table A in S5 Table), while the S000453 is the salinity-induced element [46] presented in the regulated genes in both of roots and leaves exposed to salinity stress (Table B in S5 Table). In addition, we predicted the new cis-elements of the JcNAC genes' promoters using MEME program (S5 Table). It will be helpful for us to further study on the expression regulation of the JcNAC genes in future. 

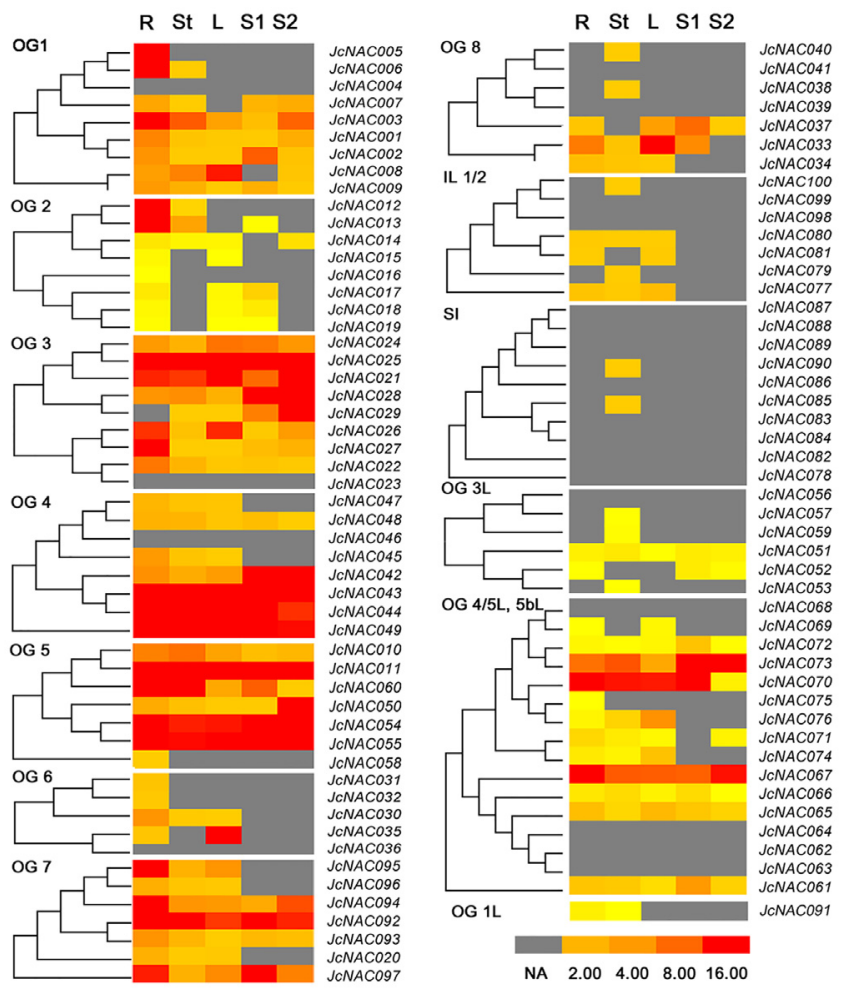

Fig 4. Relative expression levels of JcNAC genes, divided into different orthologous groups. The relative expression levels of all of the JCNAC genes were the average value of 6 biological repeats of digital expression profile tags. NA, not available; R, roots; St, stem cortex; L, leaves; S1, seed 1; S2, seed 2.

doi:10.1371/journal.pone.0131890.g004

\section{Discussion}

The NAC family genes have been classified into two major groups, Groups I and II, by comprehensive analysis of NAC sequences, and those of Group I (but not Group II) are highly conserved [7]. Group I and some Group II sequences have been further classified into 40 orthologous groups (OGs), which probably derived from a gene present in the most recent common ancestor of dicots and monocots, according to an expert NAC sequence comparison [20]. In this study, we identified a total of 100 NAC genes (JcNACs) in the physic nut genome (Table 1). After testing the predicted motifs from the JcNAC proteins, we observed that one or more motifs of the NAC domain in $40 \%$ of JcNAC proteins have diverged or been lost (Fig 1), which may have resulted in their functional divergence or nonfunctionalization. According to exon/intron structure (S2 Table) and phylogenetic analyses (S2 and S3 Figs), 60 JcNAC proteins clustered into the 39 proposed OGs for dicots, with one to five members in each OG (Table 1). These findings indicate that all the detected OGs of dicots' NAC genes but OG3b have been retained in the physic nut genome.

The 15 OG5b-like (5bL) genes are close to the OG5b clade in the phylogenetic tree. Gene expansion in this group has mainly arisen from tandem duplication events. T5 on chromosome 6 and T7 on chromosome 8 contain 11 bL tandem gene repeats (Fig 2). Nine 5bL genes cluster within the T7 locus and this duplication is also present in the castor bean genome (Fig 3 and S2 Table). Genes within this locus appear to have lost both exons and introns during their evolutionary history (Fig 1 and S2 Table). The grape genome contains multi-copy NAC genes in OG5b, mostly clustered on chromosome 15 (from 16, $45 \mathrm{Mbp}$ to 16, $51 \mathrm{Mbp}$ ). This locus has at least nine NAC genes or pseudogenes, including VvNAC50-55 (from GSVIVT01027467001 to 

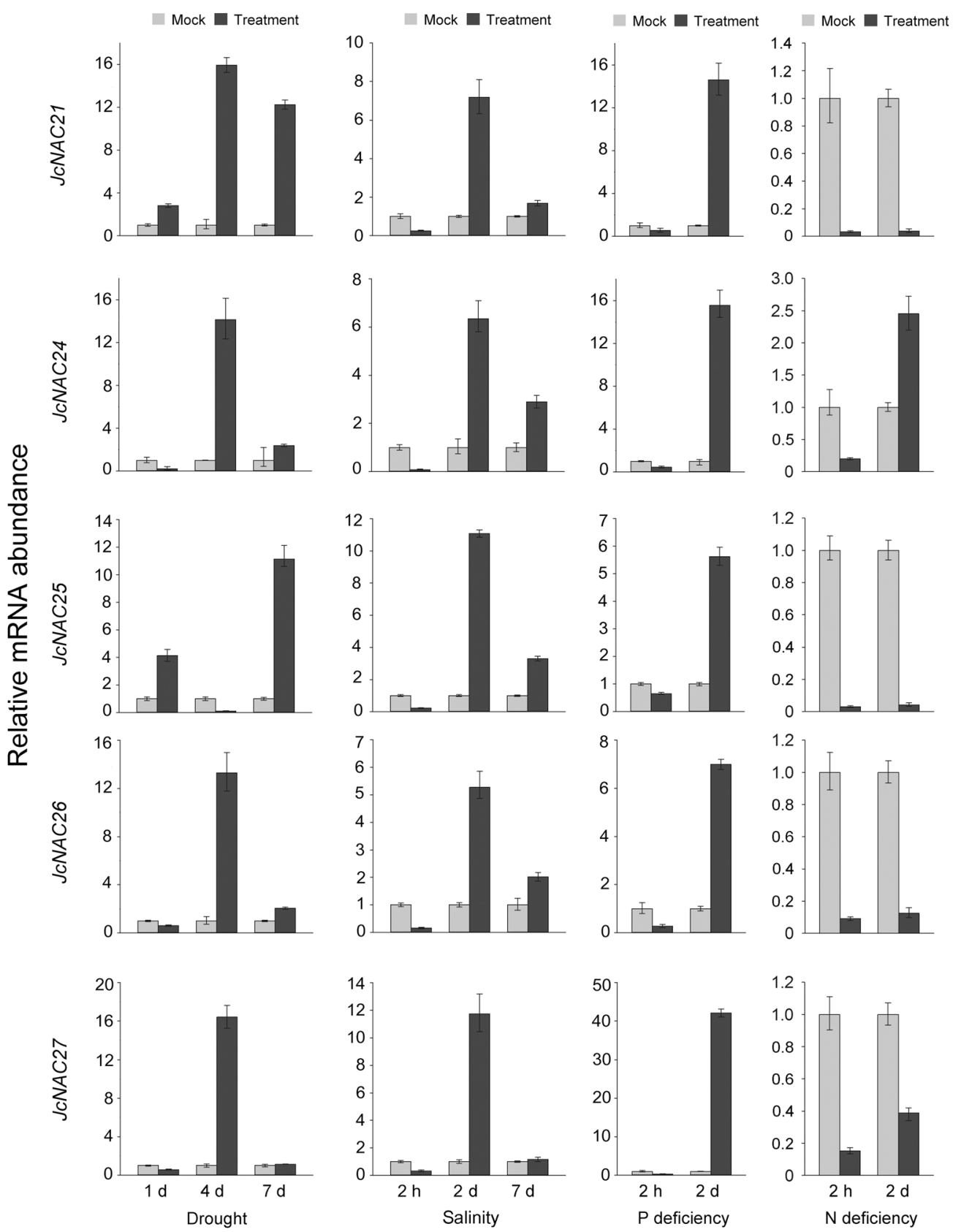

Fig 5. Expression analysis of selected JcNAC genes. Results of quantitative PCR analysis of transcripts in roots, the expression level of each gene under common condition sampled as mock. The relative expression was normalized to the reference gene JcActin as an internal control. The bars show standard deviations of the repeats. Each assay was run in triplicate for two independent biological repeats.

doi:10.1371/journal.pone.0131890.g005

GSVIVT01027477001, http://www.phytozome.net/) [15], and two unannotated NAC genes between GSVIVT01027467001 and GSVIVT01027470001. Two tandem NAC genes were annotated as a single gene of VvNAC54 (GSVIVT01027475001). These results strongly suggest that $5 \mathrm{bL}$ genes in physic nut and castor bean evolved from OG5b genes, so we named them OG5-like (5bL). The 5bL subgroup is phylogenetically close to ANAC001 subgroup [7], which is existed in the Populus trichocarpa genome (within the NAC-k subgroup) [40] but not in the rice genome 
(Panel G in S3 Fig). Thirteen NAC proteins in Arabidopsis and six in rice contain strong $\alpha$-helical transmembrane motifs in their C-terminus regions, some of which are involved in stress responses and phytohormone-mediated signal pathways in Arabidopsis [47, 48]. The genes from Arabidopsis and rice were distributed in OGs 4d (2 (in Arabidopsis), 2 (in rice)), 4g (3,1), 5b $(3,2)$, and 5c $(2,1)$. The other three Arabidopsis genes, ANAC001 (AT1G01010), ANAC068 (AT4G01540), and ANAC069 (AT4G01550), belong to the ANAC001 subgroup [7]. ANAC067069 are tandem duplications. ANAC067 has lost the transmembrane motif, and may be a pseudogene [49]. Eight JcNAC proteins containing transmembrane motifs at the C-terminus were detected (S2 Table), and they were distributed in OGs 4a (1), 4d (1), 4g (1), 5b (2), 5c (1), and 5bL (2). Physic nut has two OG4d genes: JcNAC043 and JcNAC044. The JcNAC043 gene has lost two exons in its 3'-terminus, which encode the $\alpha$-helical transmembrane motif in the JcNAC044 gene (S2 Table). The gain and/or loss of different exons in the 3'-terminus of genes in OGs 4 and 5 has probably resulted in the gain or loss of $\alpha$-helical transmembrane motifs in proteins encoded by these OGs during their evolution. Loss of the transmembrane motif in duplicated genes may result in functional divergence or pseudogenization in plants.

Twenty-three JcNAC proteins were assigned to four OGs. The six OG3-like (3L) genes are phylogenetically close to the OG3 clade, have higher amino acid identities in the NAC domain with OG3 proteins than other OG proteins, and the same exon/intron structure as OG3 genes. So, we propose they have the same origin as OG3 genes. The origins of JcNAC061 and JcNAC091 are uncertain, and they were named OGs 4, 5-like (4/5L) and OG1-like (1L), mainly based on their exon/intron structures and the phylogenetic analysis. The orthologs of $3 \mathrm{~L}$ genes were detected in subgroups of NAC- $j$ and NAC-m, while orthologs of $1 \mathrm{~L}$ genes were in the NAC-n subgroup of Populus NAC genes [40]. The ortholog of 4/5L genes was detected in the genome of T. cacao (its locus names in Phytozome (http://www.phytozome.net/) is Thecc1EG033799), but not in Populus, Arabidopsis and rice. On the other hand, a number of NAC genes containing one of two introns within their NAC domain coding sequences in Arabidopsis and rice were unclassified into the determined OGs (Panel G in S3 Fig). The amino acid sequences of their NAC domains are highly divergent from other subgroup NAC proteins, and their orthologs were not found in the physic nut genome. These results indicate that different plant linkages may have either lost or acquired some of the NAC subfamilies during their evolution. Among these genes, only ANAC095 (AT5G41090.1) was reported to play a role in male gametophyte development in Arabidopsis as yet [50].

Intronless (IL) NAC genes were found in all examined plants, and they fall into the previously reported Group II [7]. The IL genes from different species were clustered into distinct subgroups among different plant groups (S2 Fig and Panel G in S3 Fig). Seven IL NAC genes were detected in physic nut, and 13 in castor bean. Most of them are tandem duplications. In physic nut, the IL genes are clustered in T2 and T10 on chromosomes 4 and 10 (Fig 2), while scaffold 29657 of castor bean contains a cluster of six IL NAC genes. Arabidopsis also has a cluster of five IL NAC genes (AT1G60280, AT1G60300, AT1G60340, AT1G60350, and $A T 1 G 60380$ ). The origin of these IL NAC genes is unclear because their NAC domains appear to be very divergent from the other NACs and they do not have conserved amino acid sequences among different plant groups. Furthermore, little is known about their functions in plants as yet.

The only plant species of the seven examined (physic nut, Arabidopsis, castor bean, grape, rice, poplar, and soybean) in which single intron-containing (SI) genes were detected was physic nut. In this group, NAC subdomains A-D are encoded by the first exon, and subdomain $\mathrm{E}$ by the second exon (S2 Table). Using these proteins as queries in BlastP searches against public protein databases, we found orthologs in the T. cacao genome (Thecc1EG022059, Thecc1EG030287, Thecc1EG036193, Thecc1EG036197, and Thecc1EG036236). These results 
indicate that many plant species have lost SI genes during their evolution. Their origin is also unclear because their NAC domains appear to be very divergent from those of other NACs.

The segmental duplication putatively associated with the salicoid genomic duplication event appears to have significantly contributed to the amplification of many multi-gene families [31]. Accordingly, our phylogenetic analysis and chromosomal mapping indicate that segmental duplication blocks (A) contain some of the duplicated genes (of the same OGs) in the physic nut genome (Fig 2) based on the corresponding duplicate genomic blocks established in this species [26], e.g. OGs $3 \mathrm{c}$ and $8 \mathrm{~b}$ in A1 blocks, OG7a in A2 blocks, OG3c and 5bL in A3 blocks, $5 \mathrm{bL}$ in A4 blocks, and SI in A5 blocks. Tandem duplications may also have affected expansion of the NAC gene family [14-16, 40]. The gene loci analysis revealed the presence of 11 tandem duplicate clusters (T), including $30 \%$ of the NAC genes in the physic nut genome. Genes in eight of these clusters were classified into the same OGs. However, three of these tandem duplications could be ancient, resulting in divisions of the duplicated genes into different clades in the phylogenetic tree. As mentioned above, several tandem duplicates in the T7 locus appear to have lost or gained exons, and may have undergone pseudogenization like their orthologs in Arabidopsis [49]. The T6 tandem duplicates probably arose from an ancient duplication event preceding the divergence of monocots and dicots [20]. T1 tandem duplications likely arose from a dicot-specific event that divided OGs $3 e$ and $3 \mathrm{~d}$, as OG3e has not been detected in monocots (Table 1; Fig 2 and S2 Fig). Only T4, T8, T9, and T11 tandem duplications likely occurred after separation of lineages of the analyzed species (Fig 2). Additionally, several duplicates from the ancient duplication events (A1, A2, and A3) and tandem duplication events (T7 and T11) show divergent expression patterns (Fig 4 and S4 Table), suggesting the occurrence of subfunctionalization during the evolutionary process.

The function of NAC genes in most OGs has been addressed in Arabidopsis and/or other plants (S3 Table) [20]. We found that many NAC orthologs of physic nut and Arabidopsis have similar tissue-specific, and abiotic stress response, expression patterns (S3 Table). For example, the OG3d genes ATNAC2/ANAC056 and ANAC018 in Arabidopsis participate in regulation of embryogenesis [51]; while in physic nut $J c N A C 028$ and 029 are only highly expressed in seeds. Most ANAC genes in OG3 respond to abiotic stresses in Arabidopsis [39]. For instance, the OG3d gene ATNAC2 in Arabidopsis reportedly responds to salinity stress, but not drought stress [49], and the same responses were observed for JcNAC028 and 029 in physic nut roots (S3 Table). The expression levels of OG3e genes (ANAC01, ATNAC3/ANAC055 and $J c N A C 021)$ as well as 3c genes (ANAC029, ANAC047, JcNAC026 and JcNAC027) are upregulated under drought and salinity stresses in both Arabidopsis [52] and physic nut (Fig 5 and S4 Table). Expression levels of OG3a genes (ATAF1/ANAC002) are upregulated under both drought and salinity stresses and play negative roles in abiotic stress resistance Arabidopsis [53, 54]. The expression of $J c N A C 021,024,026$ and 027 are also reportedly upregulated under salinity stress in roots and leaves of physic nut seedlings [23]. These results imply that most JcNAC genes in physic nut have similar functions to their orthologs in Arabidopsis, but several may have functionally diverged from their orthologs.

In addition, the expression of $J C N A C 021,024,025,026$ and 027 respond to phosphorus and nitrogen deficiency (Fig 5). There are few reports on responses to this stress in other species. So these results may help efforts to identify further roles of NAC family members. No credible expression of the SI genes was observed in physic nut based on the available transcript sequences and next-generation sequencing-based digital gene expression tags. However, they may be expressed in other tissues (e.g. ESTs representing four orthologous SI NAC genes appear to be expressed in J. integerrima flowers), or some of them may be pseudogenes.

In conclusion, we identified a total of $100 J c N A C$ genes in the physic nut genome. Sixty of the proteins they putatively encode were grouped into 39 previously predicted OGs of NAC 
gene family. About $30 \%$ of the JcNAC genes were replicates, clustered at several locations in the physic nut genome, apparently originating from both ancient and recent duplication events.

Tissue-specific expression profiles of JcNAC transcripts under normal growth conditions were examined, and $29 J c N A C$ genes were also found to respond to tested abiotic stresses. The new $J c N A C$ gene sequences and expression information reported here should facilitate further investigations of the functions of NAC genes in plant development and stress responses, particularly in physic nut.

\section{Supporting Information}

S1 Fig. Motif logos and subdomain compositions of JcNAC proteins. Motif logos were obtained from MEME (http://meme.nbcr.net/meme/) [35]. The height of a letter indicates its relative frequency at the given position. Motifs 1 plus $8,4,6$ plus 2, 5 plus 3 , and 7 were identified as NAC subdomains A to E, respectively. (TIF)

S2 Fig. Maximum likelihood phylogenetic tree of NAC proteins. Phylogenetic analysis was conducted with protein sequences from A. thaliana, O. sativa, R. communis and J. curcas. Branch support values correspond to approximate likelihood ratio test (a-LRT) results. Bootstrap scores higher than $50 \%$ are indicated on the nodes. The 40 distinct orthologous groups (OGs) are indicated by blue characters with the names assigned by Cenci et al. [20]. We named the OGs marked by green characters. Detailed grouping information is given in S3 Table. (TIF)

S3 Fig. Maximum likelihood phylogenetic trees of each main cluster of NAC proteins. (PDF)

S1 Table. Primers used for qRT-PCR.

S2 Table. Overview of the JcNAC genes.

S3 Table. List of the NAC gene family based on phylogenetic analysis in this study. (XLS)

S4 Table. The expression of JcNAC genes based on digital gene expression analysis. (XLS)

S5 Table. Putative cis-acting elements enriched in promoters of $J c N A C$ genes exposed to four different stresses.

(XLS)

\section{Author Contributions}

Conceived and designed the experiments: HJ GW ML. Performed the experiments: ZW XX WX. Analyzed the data: ZW PW YC. Contributed reagents/materials/analysis tools: ZW XX WX PW. Wrote the paper: ZW.

\section{References}

1. Souer E, vanHouwelingen A, Kloos D, Mol J, Koes R. The No Apical Meristem gene of petunia is required for pattern formation in embryos and flowers and is expressed at meristem and primordia boundaries. Cell. 1996; 85: 159-170. doi: 10.1016/S0092-8674(00)81093-4 PMID: 8612269 
2. Aida M, Ishida T, Fukaki H, Fujisawa H, Tasaka M. Genes involved in organ separation in Arabidopsis: an analysis of the cup-shaped cotyledon mutant. Plant Cell. 1997; 9: 841-857. doi: 10.1105/tpc.9.6. 841 PMID: 9212461

3. Delessert C, Kazan K, Wilson IW, Van Der Straeten D, Manners J, Dennis ES, et al. The transcription factor ATAF2 represses the expression of pathogenesis-related genes in Arabidopsis. Plant J. 2005; 43: 745-757. doi: 10.1111/j.1365-313x.2005.02488.x PMID: 16115070

4. Wu Y, Deng Z, Lai J, Zhang Y, Yang C, Yin B, et al. Dual function of Arabidopsis ATAF1 in abiotic and biotic stress responses. Cell Res. 2009; 19: 1279-1290. doi: 10.1038/cr.2009.108 PMID: 19752887

5. Kikuchi K, Ueguchi-Tanaka M, Yoshida KT, Nagato Y, Matsusoka M, Hirano HY. Molecular analysis of the NAC gene family in rice. Mol Gen Genet. 2000; 262: 1047-1051. doi: 10.1007/pl00008647 PMID: 10660065

6. Duval M, Hsieh TF, Kim SY, Thomas TL. Molecular characterization of AtNAM: a member of the Arabidopsis NAC domain superfamily. Plant Mol Biol. 2002; 50: 237-248. doi: 10.1023/a:1016028530943 PMID: 12175016

7. Ooka H, Satoh K, Doi K, Nagata T, Otomo Y, Murakami K, et al. Comprehensive analysis of NAC family genes in Oryza sativa and Arabidopsis thaliana. DNA Res. 2003; 10: 239-247. doi: 10.1093/dnares/10. 6.239 PMID: 15029955

8. Sablowski RWM, Meyerowitz EM. A homolog of NO APICAL MERISTEM is an immediate target of the floral homeotic genes APETALA3/PISTILLATA. Cell. 1998; 92: 93-103. doi: 10.1016/s0092-8674(00) 80902-2 PMID: 9489703

9. Yamaguchi M, Ohtani M, Mitsuda N, Kubo M, Ohme-Takagi M, Fukuda H, et al. VND-INTERACTING2, a NAC domain transcription factor, negatively regulates xylem vessel formation in Arabidopsis. Plant Cell. 2010; 22: 1249-1263. doi: 10.1105/tpc.108.064048 PMID: 20388856

10. Guo YF, Gan SS. AtNAP, a NAC family transcription factor, has an important role in leaf senescence. Plant J. 2006; 46: 601-612. doi: 10.1111/j.1365-313x.2006.02723.x PMID: 16640597

11. Shan W, Kuang JF, Chen L, Xie H, Peng HH, Xiao YY, et al. Molecular characterization of banana NAC transcription factors and their interactions with ethylene signalling component EIL during fruit ripening. J Exp Bot. 2012; 63: 5171-5187. doi: 10.1093/jxb/ers178 PMID: 22888129

12. Bu QY, Jiang HL, Li CB, Zhai QZ, Zhang JY, Wu X, et al. Role of the Arabidopsis thaliana NAC transcription factors ANAC019 and ANAC055 in regulating jasmonic acid-signaled defense responses. Cell Res. 2008; 18: 756-767. doi: 10.1038/cr.2008.53 PMID: 18427573

13. Hao YJ, Wei W, Song QX, Chen HW, Zhang YQ, Wang F, et al. Soybean NAC transcription factors promote abiotic stress tolerance and lateral root formation in transgenic plants. Plant J. 2011; 68: 302313. doi: 10.1111/j.1365-313x.2011.04687.x PMID: 21707801

14. Nuruzzaman M, Manimekalai R, Sharoni AM, Satoh K, Kondoh H, Ooka H, et al. Genome-wide analysis of NAC transcription factor family in rice. Gene. 2010; 465: 30-44. doi: 10.1016/j.gene.2010.06.008 PMID: 20600702

15. Wang N, Zheng Y, Xin HP, Fang LC, Li SH. Comprehensive analysis of NAC domain transcription factor gene family in Vitis vinifera. Plant Cell Rep. 2013; 32: 61-75. doi: 10.1007/s00299-012-1340-y PMID: 22983198

16. Le DT, Nishiyama R, Watanabe Y, Mochida K, Yamaguchi-Shinozaki K, Tran LS. Genome-wide survey and expression analysis of the plant-specific NAC transcription factor family in soybean during development and dehydration stress. DNA Res. 2011; 18: 263-276. doi: 10.1093/dnares/dsr015 PMID: 21685489

17. Satheesh $V$, Jagannadham $P T K$, Chidambaranathan $P$, Jain $P K$, Srinivasan $R$. NAC transcription factor genes: genome-wide identification, phylogenetic, motif and cis-regulatory element analysis in pigeonpea (Cajanus cajan (L.) Millsp.). Mol Biol Rep. 2014; 41: 7763-7773. doi: 10.1007/s11033-0143669-5 PMID: 25108674

18. Puranik S, Sahu PP, Mandal SN, B VS, Parida SK, Prasad M. Comprehensive genome-wide survey, genomic constitution and expression profiling of the NAC transcription factor family in foxtail millet (Setaria italica L.). PLoS ONE. 2013; 8: e64594. doi: 10.1371/journal.pone.0064594 PMID: 23691254

19. Liu TK, Song XM, Duan WK, Huang ZN, Liu GF, Li Y, et al. Genome-wide analysis and expression patterns of NAC transcription factor family under different developmental stages and abiotic stresses in Chinese cabbage. Plant Mol Biol Rep. 2014; 32: 1041-1056. doi: 10.1007/s11105-014-0712-6

20. Cenci A, Guignon V, Roux N, Rouard M. Genomic analysis of NAC transcription factors in banana (Musa acuminata) and definition of NAC orthologous groups for monocots and dicots. Plant Mol Biol. 2014; 85: 63-80. doi: 10.1007/s11103-013-0169-2 PMID: 24570169 
21. King AJ, He W, Cuevas JA, Freudenberger M, Ramiaramanana D, Graham IA. Potential of Jatropha curcas as a source of renewable oil and animal feed. J Exp Bot. 2009; 60: 2897-2905. doi: 10.1093/ jxb/erp025 PMID: 19218317

22. Wang $\mathrm{H}$, Zou Z, Wang S, Gong M. Global analysis of transcriptome responses and gene expression profiles to cold stress of Jatropha curcas L. PLoS ONE. 2013; 8: e82817. doi: 10.1371/journal.pone. 0082817 PMID: 24349370

23. Zhang $\mathrm{L}$, Zhang $\mathrm{C}$, Wu $\mathrm{P}$, Chen $\mathrm{Y}$, Li M, Jiang $\mathrm{H}$, et al. Global analysis of gene expression profiles in physic nut (Jatropha curcas L.) seedlings exposed to salinity stress. PLoS ONE. 2014; 9: e97878. doi: 10.1371/journal.pone.0097878 PMID: 24837971

24. Sato S, Hirakawa $H$, Isobe $S$, Fukai $E$, Watanabe $A$, Kato $M$, et al. Sequence analysis of the genome of an oil-bearing tree, Jatropha curcas L. DNA Res. 2011; 18: 65-76. doi: 10.1093/dnares/dsq030 PMID: 21149391

25. Eswaran N, Parameswaran S, Anantharaman B, Kumar GRK, Sathram B, Johnson TS. Generation of an expressed sequence tag (EST) library from salt-stressed roots of Jatropha curcas for identification of abiotic stress-responsive genes. Plant Biol. 2012; 14: 428-437. doi: 10.1111/j.1438-8677.2011. 00529.x PMID: 22329502

26. Wu P, Zhou C, Cheng S, Wu Z, Lu W, Han J, et al. Integrated genome sequence and linkage map of physic nut (Jatropha curcas L.), a biodiesel plant. Plant J. 2015; 81: 810-821. doi: 10.1111/tpj.12761 PMID: 25603894

27. Xiong WD, Xu XQ, Zhang L, Wu PZ, Chen YP, Li M, et al. Genome-wide analysis of the WRKY gene family in physic nut (Jatropha curcas L.). Gene. 2013; 524 (2): 124-132. doi: 10.1016/j.gene.2013.04. 047 PMID: 23644253

28. Bjellqvist B, Basse B, Olsen E, Celis JE. Reference points for comparisons of two-dimensional maps of proteins from different human cell types defined in a $\mathrm{pH}$ scale where isoelectric points correlate with polypeptide compositions. Electrophoresis. 1994; 15: 529-539. doi: 10.1002/elps.1150150171 PMID: 8055880

29. Voorrips RE. MapChart: Software for the graphical presentation of linkage maps and QTLs. J Hered. 2002; 93: 77-78. doi: 10.1093/jhered/93.1.77 PMID: 12011185

30. Cannon SB, Mitra A, Baumgarten A, Young ND, May G. The roles of segmental and tandem gene duplication in the evolution of large gene families in Arabidopsis thaliana. BMC Plant Biol. 2004; 4: 10. doi: 10.1186/1471-2229-4-10 PMID: 15171794

31. Goodstein DM, Shu SQ, Howson R, Neupane R, Hayes RD, Fazo J, et al. Phytozome: a comparative platform for green plant genomics. Nucleic Acids Res. 2012; 40: D1178-1186. doi: 10.1093/nar/gkr944 PMID: 22110026

32. Guindon S, Dufayard JF, Lefort V, Anisimova M, Hordijk W, Gascuel O. New algorithms and methods to estimate maximum-likelihood phylogenies: assessing the performance of PhyML 3.0. Syst Biol. 2010; 59: 307-321. doi: 10.1093/sysbio/syq010 PMID: 20525638

33. Guo AY, Zhu QH, Chen X, Luo JC. GSDS: a gene structure display server. Yi Chuan. 2007; 29: 10231026. doi: 10.1360/yc-007-1023 PMID: 17681935

34. Bailey TL, Boden M, Buske FA, Frith M, Grant CE, Clementi L, et al. MEME SUITE: tools for motif discovery and searching. Nucleic Acids Res. 2009; 37: 202-208. doi: 10.1093/nar/gkp335 PMID: 19458158

35. Krogh A, Larsson B, von Heijne G, Sonnhammer EL. Predicting transmembrane protein topology with a hidden Markov model: application to complete genomes. J Mol Biol. 2001; 305: 567-580. doi: 10.1006/ jmbi.2000.4315 PMID: 11152613

36. Higo K, Ugawa Y, Iwamoto M, Korenaga T. Plant cis-acting regulatory DNA elements (PLACE) database: 1999. Nucleic Acid Res. 1999; 27: 297-300. doi: 10.1093/nar/27.1.297 PMID: 9847208

37. Chang S, Puryear J, Cairney J. A simple and efficient method for isolating RNA from pine trees. Plant Mol Biol Rep. 1993; 11: 113-116. doi: 10.1007/bf02670468

38. Singh AK, Sharma V, Pal AK, Acharya V, Ahuja PS. Genome-wide organization and expression profiling of the NAC transcription factor family in potato (Solanum tuberosum L.). DNA Res. 2013; 20: 403423. doi: 10.1093/dnares/dst019 PMID: 23649897

39. Puranik S, Sahu PP, Srivastava PS, Prasad M. NAC proteins: regulation and role in stress tolerance. Trends Plant Sci. 2012; 17: 369-381. doi: 10.1016/j.tplants.2012.02.004 PMID: 22445067

40. Hu RB, Qi G, Kong YZ, Kong DJ, Gao Q, Zhou G. Comprehensive analysis of NAC domain transcription factor gene family in Populus trichocarpa. BMC Plant Biol. 2010; 10: 145. doi: 10.1186/1471-222910-145 PMID: 20630103 
41. Jiang HW, Wu PZ, Zhang S, Song C, Chen YP, Li M, et al. Global analysis of gene expression profiles in developing physic nut (Jatropha curcas L.) seeds. PLoS ONE. 2012; 7: e36522. doi: 10.1371/ journal.pone.0036522 PMID: 22574177

42. Zhang $C$, Zhang L, Zhang S, Zhu S, Wu P, Chen Y, et al. Global analysis of gene expression profiles in physic nut (Jatropha curcas L.) seedlings exposed to drought stress. BMC Plant Biol. 2015; 15: 17. doi: 10.1186/s12870-014-0397-x PMID: 25604012

43. Audic S, Claverie JM. The significance of digital gene expression profiles. Genome Res. 1997; 7: 986995. doi: 10.1101/gr.7.10.986 PMID: 9331369

44. Romualdi C, Bortoluzzi S, D'Alessi F, Danieli GA. IDEG6: a web tool for detection of differentially expressed genes in multiple tag sampling experiments. Physiol Genomics. 2003; 12: 159-162. doi: 10. 1152/physiolgenomics.00096.2002 PMID: 12429865

45. Abe H, Urao T, Ito T, Seki M, Shinozaki K, Yamaguchi-Shinozaki K. Arabidopsis AtMYC2 (bHLH) and AtMYB2 (MYB) function as transcriptional activators in abscisic acid signaling. Plant Cell. 2003; 15: 63-78. doi: 10.1105/tpc.006130 PMID: 12509522

46. Park HC, Kim ML, Kang YH, Jeon JM, Yoo JH, Kim MC, et al. Pathogen- and NaCl-induced expression of the SCaM-4 promoter is mediated in part by a GT-1 box that interacts with a GT-1-like trancription factor. Plant Physiol. 2004; 135: 2150-2161. doi: 10.1104/pp.104.041442 PMID: 15310827

47. Kim SY, Kim SG, Kim YS, Seo PJ, Bae M, Yoon HK, et al. Exploring membrane-associated NAC transcription factors in Arabidopsis: implications for membrane biology in genome regulation. Nucleic Acids Res. 2007; 35: 203-213. doi: 10.1093/nar/gkl1068 PMID: 17158162

48. Kim YS, Park CM. Membrane regulation of cytokinin-mediated cell division in Arabidopsis. Plant Signal Behav. 2007; 2: 15-16. doi: 10.4161/psb.2.1.3606 PMID: 19704799

49. Kim YS, Kim SG, Park JE, Park HY, Lim MH, Chua NH, et al. A membrane-bound NAC transcription factor regulates cell division in Arabidopsis. Plant Cell. 2006; 18: 3132-3144. doi: 10.1105/tpc.106. 043018 PMID: 17098812

50. Reňák $D$, Dupl'áková $N$, Honys $D$. Wide-scale screening of T-DNA lines for transcription factor genes affecting male gametophyte development in Arabidopsis. Sex Plant Reprod. 2012; 25: 39-60. doi: 10. 1007/s00497-011-0178-8 PMID: 22101548

51. Kunieda T, Mitsuda N, Ohme-Takagi M, Takeda S, Aida M, Tasaka M, et al. NAC family proteins NARS1/NAC2 and NARS2/NAM in the outer integument regulate embryogenesis in Arabidopsis. Plant Cell. 2008; 20: 2631-2642. doi: 10.1105/tpc.108.060160 PMID: 18849494

52. Fujita $Y$, Fujita M, Shinozaki K, Yamaguchi-Shinozaki K. ABA-mediated transcriptional regulation in response to osmotic stress in plants. J Plant Res. 2011; 124: 509-525. doi: 10.1007/s10265-011. 0412-3 PMID: 21416314

53. Matsui A, Ishida J, Morosawa T, Mochizuki Y, Kaminuma E, Endo TA, et al. Arabidopsis transcriptome analysis under drought, cold, high-salinity and $A B A$ treatment conditions using a tiling array. Plant Cell Physiol. 2008; 49: 1135-1149. doi: 10.1093/pcp/pcn101 PMID: 18625610

54. Lu PL, Chen NZ, An R, Su Z, Qi BS, Ren F, et al.-A novel drought-inducible gene, ATAF1, encodes a NAC family protein that negatively regulates the expression of stress-responsive genes in Arabidopsis. Plant Mol Biol. 2007; 63: 289-305. doi: 10.1007/s11103-006-9089-8 PMID: 17031511 